\title{
Atenuação dos efeitos dos problemas inversos de transferência de calor pelo método dos mínimos quadrados simplificado
}

\author{
Priscila Marques da Paz Juliana de Oliveira \\ Departamento de Ciências Biológicas, Faculdade de Ciências e Letras, UNESP \\ CEP 19806-900, ASSIS, SP \\ E-mail:pri_marques_paz@hotmail.com_ juliana@assis.unesp.br
}

\begin{abstract}
RESUMO
Transferência de calor é a ciência que estuda eventos relacionados à troca de energia entre corpos, resultante da diferença de temperatura existente entre eles. Nos processos térmicos industriais, a temperatura é uma das variáveis físicas mais avaliadas, permitindo que a transferência energética seja detectada por três mecanismos distintos: condução, convecção e radiação. A transferência de calor referente ao transporte de carga térmica entre um fluido e um sólido denomina-se convecção, e pode ser combinada com os efeitos da condução e radiação [1] [3]. Esses três fenômenos requerem que técnicas de medição, monitoramento e controle sejam estabelecidas, garantindo assim a qualidade operacional dos sistemas em que eles ocorrem [1]. A determinação adequada das propriedades de transferência de calor recebe auxílio dos problemas inversos que se caracterizam por serem mal condicionados, pois pequenos erros experimentais nos dados de entrada trazem instabilidade ao sistema inverso [3].

Considerando convecção e radiação existentes no processo, o que tornam o modelo matemático inverso mais problemático, o objetivo deste trabalho é determinar uma faixa de atuação para o coeficiente de convecção, aplicando-se a técnica de regularização proposta em [1], e assim atenuar a amplificação dos ruídos indesejáveis inerentes à aquisição da temperatura.

A metodologia adotada para o sistema de monitoramento do coeficiente de convecção, baseada em um processo de escoamento, consiste na obtenção de temperaturas através da medição de dois termopares de mesma geometria externa, mas com constantes de tempo distintas devido a diferentes encapsulamentos em suas extremidades. As temperaturas adquiridas por esses termopares constituem o processamento e resultam em um coeficiente de convecção comum entre eles.
\end{abstract}

Considerando acúmulo térmico, transferência de calor por convecção e radiação, a modelagem matemática do sistema de aquisição pode ser representada pela Equação (1):

$$
M C \frac{d T}{d t}-h A\left(T_{\infty}-T\right)-\varepsilon \sigma A\left(T_{\infty}^{4}-T^{4}\right)=0
$$

onde $M$ é a massa $(\mathrm{kg}), C$ o calor específico $(\mathrm{J} / \mathrm{KgK}), A$ a área da superfície de transferência de calor $\left(\mathrm{m}^{2}\right), h$ o coeficiente de convecção $\left(\mathrm{W} / \mathrm{m}^{2} \mathrm{~K}\right), T$ a temperatura do processo $(\mathrm{K})$ adquirida pelo termopar, $T_{\infty}$ a temperatura de escoamento livre $(\mathrm{K}), 0 \leq \varepsilon \leq 1$ o poder de emissividade da superfície, $\sigma=5,67 \times 10^{-8}$ a constante de Stefan-Boltzmann $\left(\mathrm{W} / \mathrm{m}^{2} \mathrm{~K}^{4}\right)$.

Aplicando a Equação (1) para cada um dos termopares $A$ e $B$, sendo $T_{\infty}$ o mesmo em ambas equações, é possível determinar o coeficiente de convecção comum $h$ conforme Equação (2):

$$
h=\frac{1}{A\left(T_{A}-T_{B}\right)}\left[M_{B} C_{B} \frac{d T_{B}}{d t}-M_{A} C_{A} \frac{d T_{A}}{d t}+A \varepsilon \sigma\left(T_{B}^{4}-T_{A}^{4}\right)\right]
$$

As temperaturas $A$ e $B$ indicadas pelos termopares carregam ruídos no sinal. Para atenuar os sinais obtidos, os dados são ajustados a partir do método de regularização utilizado em [1], o qual alude ao conceito dos métodos dos mínimos quadrados simplificado (MMQs). A ideia é aproximar a temperatura do processo através de um polinômio de baixo grau, poucos pontos e escolha de um peso, $T(t) \approx a_{0}+a_{1} x+a_{2} x^{2}+\cdots+a_{N} x^{N}$ e, a partir disso, obtêm-se os seguintes equacionamentos $d T / d t=a_{1}$ e $T=a_{0}$, com $a_{0}$ e $a_{1}$ calculados em tempo real. Assim, da Equação (2), o coeficiente de convecção regularizado, $h_{r e g}$, é dado pela Equação (3): 
$h_{\text {reg }}=\frac{1}{A\left(a_{0 A}-a_{0 B}\right)}\left[M_{B} C_{B} a_{1 B}-M_{A} C_{A} a_{1 A}+A \varepsilon \sigma\left(a_{0 B}^{4}-a_{0 A}^{4}\right)\right]$

O sistema foi implementado no software LabVIEWTM, sendo testado em experimentos numéricos que simularam a imersão de dois termopares numa emulsão reagente com temperatura mínima de $363 \mathrm{~K}$ e máxima de $373 \mathrm{~K}$, nos seguintes parâmetros fixos, para ambos os termopares: $M=4,7 \times 10^{-6} \mathrm{Kg}, C=3,8 \times 10^{2} \mathrm{~J} / \mathrm{KgK}$ e $A=3,14 \times 10^{-6} \mathrm{~m}^{2}$. Os testes basearam-se na aquisição de temperatura com ruídos entre $0,001 \mathrm{~K}$ a $1 \mathrm{~K}$, variando, para cada um deles, o grau do polinômio de 1 a 4, quantidade de pontos utilizada para a regularização de 10 a 20 e peso 1 .

Um dos melhores resultados observados para o coeficiente de convecção é apresentado na Figura 1. É interessante ressaltar que mesmo para ruídos altos, os quais podem descaracterizar totalmente a reconstrução da temperatura, o $h_{\text {reg }}$ ainda assim é capaz de atenuar e acompanhar o indicado $\left(h_{i n d}\right)$. Em todas as análises, foi possível manter a faixa de atuação do $h_{r e g}$ no valor absoluto de $50000 \mathrm{~W} / \mathrm{m}^{2} \mathrm{~K}$, importante na resolução de problemas detectados ao reconstruir o $h$, os quais envolvem, esporadicamente, descontinuidade e picos do sinal, mas que não comprometem o limite de atuação do $h$. Dessa maneira, os poucos valores acima dessa faixa limite são controlados e recebem o valor de $550 \mathrm{~W} / \mathrm{m}^{2} \mathrm{~K}$, que é equivalente ao do processo $\left(h_{p r o c}\right)$ [2]. O MMQs é usado apenas para a regularização da temperatura, não sendo afetado pelo controle que o $h_{\text {reg }}$ sofre, posteriormente, ao extrapolar os limites. O desempenho do monitoramento do $h$ mostra a capacidade de suavização da técnica proposta, pois $h_{\text {reg }}$ se manteve na faixa de atuação, mesmo com os coeficientes $a_{0 A}$ e $a_{0 B}$ do polinômio se apresentarem, durante a formulação (Equação (3)), à quarta potência devido à radiação.

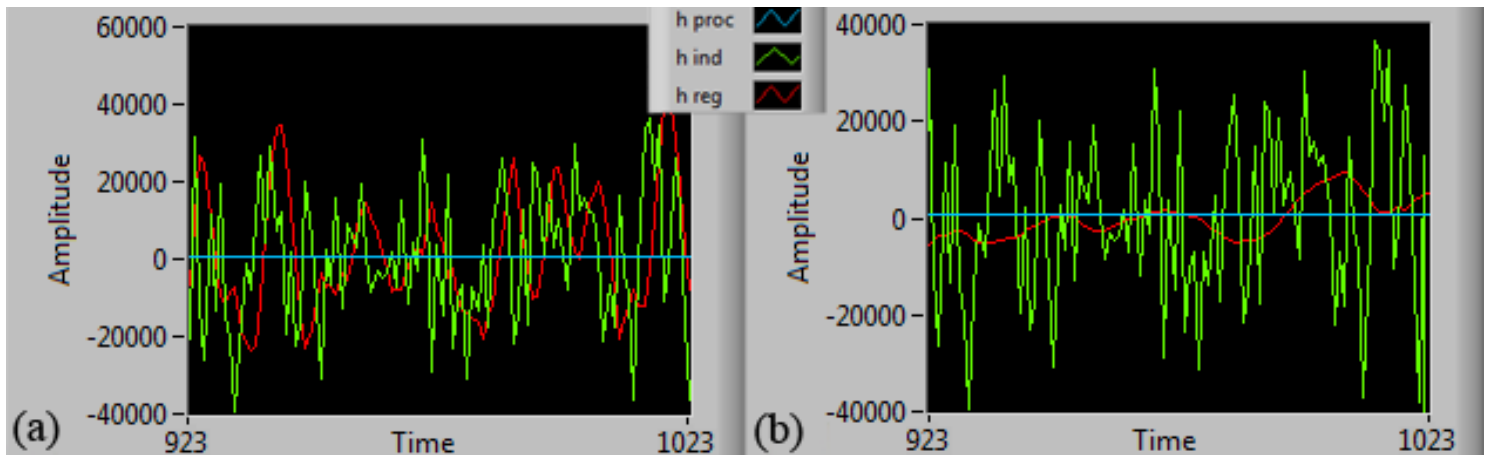

Figura 1. Sistemas de monitoramento do coeficiente de convecção para $0,01 \mathrm{~K}$ de ruído. (a) refere-se ao grau 2 do polinômio e 20 pontos de temperatura, enquanto (b), grau 1 e 20 pontos.

Portanto, conclui-se que a metodologia utilizada neste trabalho foi eficiente não apenas para delimitar uma faixa de atuação para o monitoramento do $h$, mas também mantê-lo atenuado mesmo em condições que perturbam o processo, como níveis altos de ruído e presença de radiação.

Palavras-chave: Temperatura, Convecção, Radiação, Problema inverso, Regularização.

\section{Referências}

[1] J. Oliveira, J. N. Santos e P. Seleghim Jr., Inverse measurement method for detecting bubbles in a fluidized bed reactor-toward the development of an intelligent temperature sensor, Powder Technol., vol. 169, pp. 123-135, (2006).

[2] P. M. Paz, J. Oliveira, Use of a numerical technique for monitoring of convection coefficient in industrial processes, em "22 ${ }^{\text {nd }}$ COBEM", pp.1448-1454, Ribeirão Preto, 2013.

[3] O. Wellele, H. R. B. Orlande, N. Ruperti Jr., M. J. Colaço, A. Delmas, Coupled conduction-radiation in semi-transparent materials at high temperatures. Journal of Physics and Chemistry of Solids, vol. 67, pp. 2230-2240, (2006). 